\title{
Pre-treatment evaluation of 5-fluorouracil degradation rate: association of poor and ultra-rapid metabolism with severe toxicity in a colorectal cancer patients cohort
}

\author{
Federica Mazzuca ${ }^{1,2}$, Marina Borro ${ }^{3}$, Andrea Botticelli², Eva Mazzotti ${ }^{2}$, Luca Marchetti ${ }^{4}$, \\ Giovanna Gentiles, Marco La Torre ${ }^{4}$, Luana Lionetto5, Maurizio Simmaco ${ }^{3}$, Paolo \\ Marchetti ${ }^{1,2,3}$ \\ ${ }^{1}$ Oncology Unit, Sant'Andrea Hospital, Rome, Italy \\ ${ }^{2}$ Department of Clinical and Molecular Medicine, Sapienza University of Rome, Rome, Italy \\ ${ }^{3}$ Department of Neurosciences, Mental Health and Sensory Organs (NESMOS), Sapienza University of Rome, Rome, Italy \\ ${ }^{4}$ Department of Clinical Oncology, Policlinico Umberto I, Rome, Italy \\ ${ }^{5}$ Istituto Dermopatico dell'Immacolata-IRCCS, Rome, Italy \\ Correspondence to: Eva Mazzotti, e-mail: eva.mazzotti@tiscali.it
}

Keywords: 5-fluorouracil degradation rate, fluorouracil toxicity prediction, DPYD, colorectal cancer, polymorphisms

Received: December 08, 2015

Accepted: February 15, 2016

Published: March 08, 2016

\section{ABSTRACT}

Despite the wide use of 5-fluorouracil-based chemotherapy, development of severe toxicity that follow the treatment is not a rare event. The efforts to establish pretreatment tools for toxicity prediction, led to the development of various pharmacogenetic and biochemical assays, mainly targeted to assess the activity level of dihydropyrimidine dehydrogenase (DPD), the main metabolizing enzyme for 5-fluorouracil. Using peripheral blood mononuclear cells, we developed a biochemical assay, that is not limited to the evaluation of DPD activity, but determines the net result of all the enzymatic transformation of $5 \mathrm{FU}$, in terms of the amount of drug consumed by the cells in a time unit. This parameter, named 5-fluorauracil degradation rate, presents a normal distribution inside the population and highlight the presence of an ultra-rapid metabolizers class of subjects, besides the expected poor metabolizers class. Here we will show that, in a colorectal cancer patient cohort, both poor and ultra-rapid metabolizers have significantly increased the risk of developing severe toxicity (grade3-4). Patient stratification depending on the individual 5-fluorouracil degradation rate allows to identify a $10 \%$ of the overall population at high risk of developing severe toxicity, compared to the $1.3 \%$ (as assessed in the Italian population) identified by the most commonly employed pharmacogenetic test, including the DPD polymorphism IVS14+1G >A.

\section{INTRODUCTION}

Fluorouracil, in combination with oxaliplatin, irinotecan, and biological agents, is the most common first line chemotherapy to treat colorectal cancer (CRC), both in the adjuvant and palliative setting, [1-3]. Despite efficacy, severe toxicity represents a major cause of reduced dosage, delayed drug administration and therapy discontinuation. Grade 3-4 toxicity is reported in about $30 \%$ of patients, with a $0.5 \%$ of toxic deaths $[4,5]$. This figure means that every year the 5-FU toxicity determines approximately 1,300 deaths in the USA [6] and 200 in
France or Italy [7]. Moreover, a higher number of patients suffer from unduly toxic effects, with avoidable suffering and reducible costs for the Health Systems.

Pre-emptive identification of patients who develop 5 -FU severe toxicity is still an open issue in cancer management, hence the available methods identify only a small fraction of such patients.

The dihydropyrimidine dehydrogenase enzyme (DPD, encoded by the DPYD gene) transforms about $80 \%$ of the administrated 5 -FU in the inactive metabolite 5 , 6-dihydro-5-fluorouracil. The remaining $20 \%$ of the drug is catabolized by activating enzymes (Figure 1), with 
the production of metabolites accounting for inhibition of thymidylate synthase (TYMS) and for RNA/DNA damage [8]. An impaired activity of DPD leads to an increased production of cytotoxic metabolites and has been clearly associated with 5-FU induced severe toxicities [9-11].

The most used pharmacogenetic assay to predict DPD activity evaluates the presence of the splice site IVS14+1G $>$ A polymorphism in the DPYD gene, which leads to production of a truncated, inactive protein and is associated with severe toxicity in about one half of carriers [12]. However, the IVS14+1G $>$ A polymorphism has low frequency and it is not present in the majority of the patients with high 5-FU toxicity. Recently, we described a that DPYD haplotype is associated with a decreased value of 5FUDR and it could be related to toxicity development [13]. The functional effect of additional $D P Y D$ polymorphisms has been evaluated, but for the moment their prediction power results inadequate [14].

Association with toxicity of polymorphisms in the 5-FU target TYMS and in the methylenetetrahydrofolate reductase (MTHFR) has been widely studied and these variants are frequently tested in pharmacogenetic assays, but there is not a general consensus about their clinical impact [15-18].

Along with pharmacogenetics, biochemical assays for pretreatment evaluation of 5-FU metabolism have been developed, including DPD deficiency testing in cell lysates from peripheral blood and the plasma measurement of uracil (U) and dihydrouracil/uracil ratio $\left(\mathrm{UH}_{2} / \mathrm{U}\right)$ [19-22]. The last two methods are based on the estimation of DPD activity by the level of its endogenous substrate $U$ and the resulting metabolite, $\mathrm{UH}_{2}$, in plasma. This test is characterized by good sensibility of $82,4 \%$ but low specificity of $78.4 \%$.

We previously described a pretreatment ex-vivo assay to determine the rate of peripheral blood mononuclear cells (PBMC) metabolizing 5-FU [23]. This parameter (individual 5-FU degradation rate, 5-FUDR) differs by others pretreatment assays, as it is not limited to the evaluation of DPD activity, but determines the net result of all the enzymatic transformation of 5-FU (Figure 1), in terms of the amount (nmol) of drug consumed by cells in a time unit. We also showed that the 5-FUDR value is consistently lower in patients who develop grade 3-4 toxicity [23].

The present study was aimed to evaluate the performance of 5-FUDR as a pretreatment predictor of grade 3-4 toxicity and to compare it with currently used pharmacogenetic markers. The distribution of allelic variants of the genes DPYD, TYMS and MTHFR and the pretreatment 5-FUDR was analyzed in 1010 mixed cancer patients, and the association with 5-FU toxicity was analyzed on 433 CRC patients.

\section{RESULTS}

All analyzed polymorphisms were in HardyWeinberg equilibrium [24]. In the overall population of 1010 mixed cancer patients (51.29\% females, $48.71 \%$ males, median age 66 years, age range $27-87$ ), the mean pretreatment 5-FUDR value ( \pm standard deviation, SD) was $1.54 \pm 0.41 \mathrm{ng} / \mathrm{ml} / 10^{6}$ cells $/ \mathrm{min}$, the median $1.55 \mathrm{ng} / \mathrm{ml} / 10^{6}$ cells $/ \mathrm{min}$ and the interquartile range $1.25-1.84 \mathrm{ng} / \mathrm{ml} /$ $10^{6}$ cells $/ \mathrm{min}$ (range $0.03-3.01 \mathrm{ng} / \mathrm{ml} / 10^{6}$ cells $/ \mathrm{min}$ ). The departure from a normal distribution was not statistically significant $(p=0.82)$ at all, and this result was consistent with the visual inspection of the histogram and the Kernel density curve (Figure 2). The 5-FUDR parameter is not significantly affected by age, gender, cancer type, or polymorphisms in the MTHFR and TYMS genes (Table 1). Only a small difference between mean values, at the edge of significance $(p=0.072)$, appeared for the MTHFR

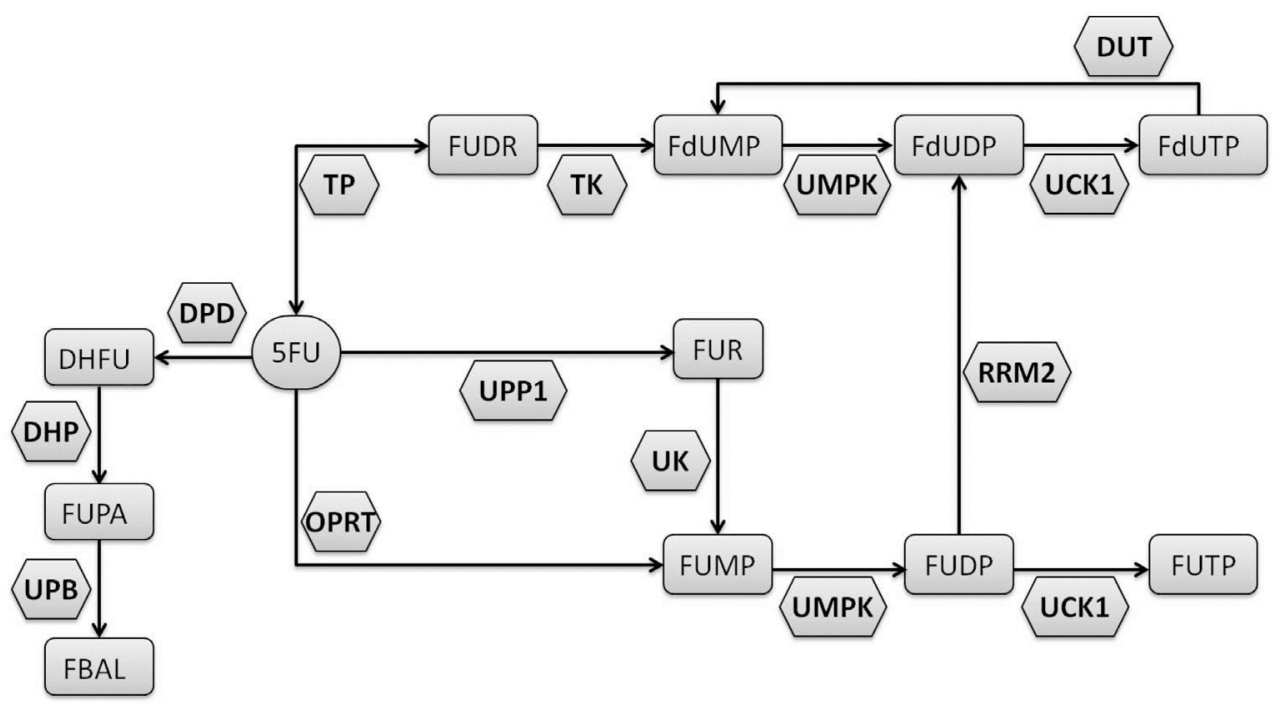

Figure 1: Metabolism of the 5-fluorouracil (5-FU). 
A1298C genotype: the homozygous carriers of the mutant $\mathrm{C}$ allele have a slight decrease in mean 5-FUDR compared to AA and AC genotypes $(p=0.072)$. In contrast, and as expected, the presence of the DPYD IVS14+1G $>$ A splice variant affects significantly $(p<0.001)$ the 5-FUDR, with the heterozygous carriers showing a marked decrease in the mean 5-FUDR value compared to non-carriers $\left(0.81 \pm 0.29 \mathrm{ng} / \mathrm{ml} / 10^{6}\right.$ cells $/ \mathrm{min}$ vs $1.54 \pm 0.41 \mathrm{ng} /$ $\mathrm{ml} / 10^{6}$ cells $/ \mathrm{min}$ ) (Table 1 ). The DPYD IVS $14+1 \mathrm{G}>\mathrm{A}$ polymorphisms was detected only as heterozygous with a frequency of $1.28 \%$.

Due to variability in chemotherapeutic regimens among different cancer types and to statistical considerations, we proceeded to study the association of 5-FUDR and gene polymorphisms with toxicity in the most numerous cancer group, that was a subset of 433 CRC patients treated with fluorouracil based regimens. Counts and frequencies of 5-FU toxicities by demographics, specific chemotherapy regimens, genetics and pre-treatment 5 -FUDR (categorized by centiles) in this subgroup are reported in Table 2. We detect a total of $92(21.2 \%)$ cases of severe toxicity (National Cancer Institute Common Toxicity Criteria, version 3 [25], CTC3, grade 3 and grade 4, G3-G4). The DPYD IVS $14+1 \mathrm{G}>\mathrm{A}$ splice variant was present as heterozygous with a frequency of $1.39 \%$, comparable to the frequency in the overall population. Of the six heterozygous carriers patients in the CRC cohort, three $(50.0 \%)$ developed severe toxicity, whereas 89 (20.8\%) out of the 427 non-carriers developed severe toxicity. The difference in number of severe toxicity events between mutated $D P Y D$ and wild-type $D P Y D$ did not reach statistical significance $(p=0.113)$. In contrast, severe toxicity was found to be significantly associated with an age above the median $(p=0.007)$ and with a 5-FUDR below the 5th centile (called poor metabolism - PM) or above the 95th centile (called ultra-rapid metabolism - UM) $(p=0.002)$. In particular, the PM and UM are associated with a 3.47 and 3.34 increased OR, respectively, compared to the normal metabolizers (NM; 5-FUDR: $0.85-2.2 \mathrm{ng} / \mathrm{ml} / 10^{6} \mathrm{cells} / \mathrm{min}$ ).
Using the 5-FUDR value as a stratification factor to identify patients at higher risk of 5-FU G3-G4 toxicity (e.g. PM or UM subjects), 40 patients (9.24\% of total population) potentially at risk can be recognized, of whom 17 (42.5\%) really developed G3-4 toxicity. Using the presence of the DPYD IVS $14+1 \mathrm{G}>\mathrm{A}$ splice variant as a risk predictive factor, we could detect only 6 patients (1.39\% of total population) carriers of the marker, of whom $3(50.0 \%)$ really developed G3-G4 toxicity. Sensitivity, specificity, positive and negative predictive values (PPV and NPV) of the DPYD IVS $14+1 \mathrm{G}>\mathrm{A}$ polymorphisms and of the 5-FUDR metabolic classes (PM, UM, and PM plus UM) are reported in Table 3.

\section{DISCUSSION}

In recent years, pharmacogenetics has been regarded as the most promising tool for preemptive risk stratification, but recent results from large studies spread some doubts about the actual usefulness of this approach. [26-28].

This is the reason why alternative roads should be walked to overcome the limits of 5-FU pharmacogenetics. 5-FU represents an elective drug to attempt biochemical approaches in predicting drug metabolism. In fact, unlike many drugs, the main metabolizing enzymes are expressed in peripheral blood cells, allowing to evaluate the efficiency of the individual drug metabolism by non-invasive procedures such as using a peripheral blood sample. Pre-treatment determination of DPD enzymatic activity in cell lysates from peripheral lymphocytes and the plasma assessment of $\mathrm{UH}_{2} / \mathrm{U}$ ratio are well established assays [7]. Previously, we developed a related biochemical assay aimed to evaluate the 5FU-metabolism preemptively, but with a significant functional difference. Whereas the above mentioned tests are targeted to determine the activity level of the DPD enzyme, the 5-FUDR assesses the combinatorial effects produced by all the 5-FU metabolizing enzymes,
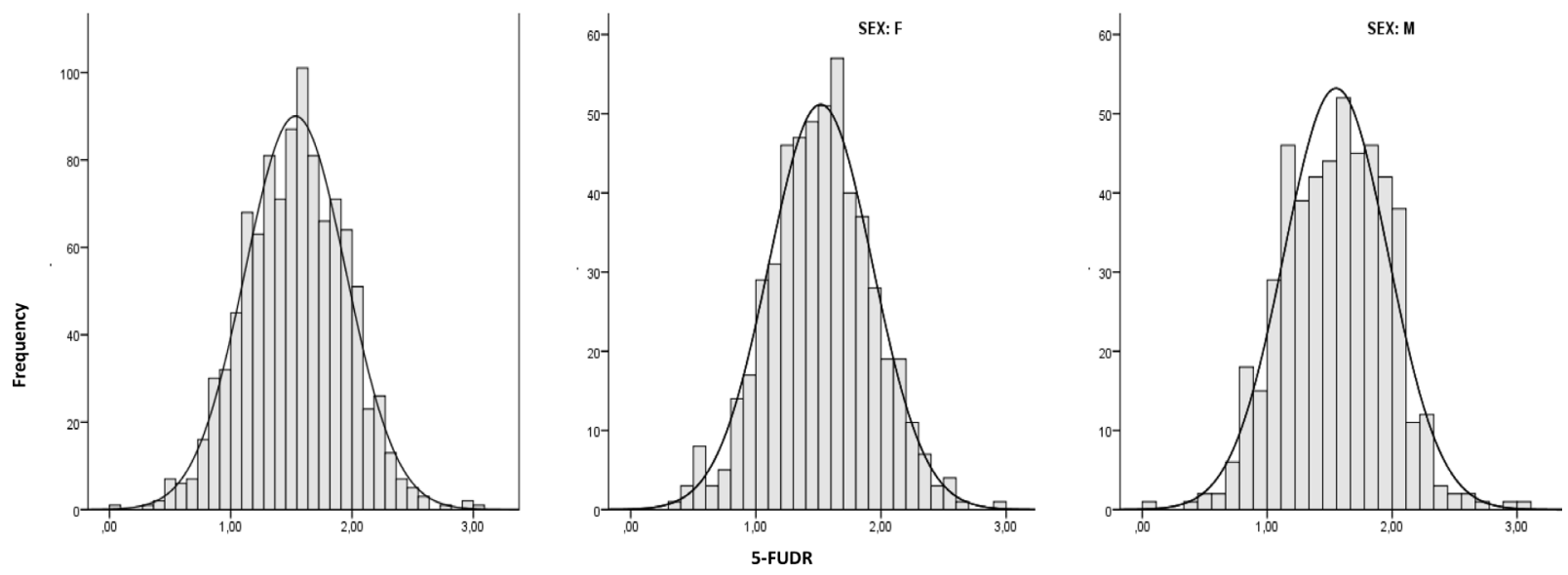

Figure 2: 5-FU degradation rate distribution $(n=1010 ; n=518$, females; $n=492$, males $)$. 
Table 1: 5-FU degradation rate (5-FUDR) means and 95\% confidence intervals by demographic, cancer type and genetic characteristics $(N=1010)$

\begin{tabular}{|c|c|c|c|c|c|c|}
\hline & & $N$ & $\%$ & mean & $95 \%$ CI & $p$-value* \\
\hline \multirow[t]{2}{*}{ Gender } & males & 492 & 48.71 & 1.55 & $1.51-1.59$ & \\
\hline & females & 518 & 51.29 & 1.52 & $1.48-1.56$ & 0.190 \\
\hline \multirow[t]{2}{*}{ Age category** } & $<=$ median & 513 & 50.79 & 1.51 & $1.47-1.55$ & \\
\hline & $>$ median & 495 & 49.01 & 1.56 & $1.52-1.60$ & 0.100 \\
\hline \multirow[t]{2}{*}{ DPYD } & GG & 998 & 98.81 & 1.54 & $1.51-1.57$ & \\
\hline & GA & 12 & 1.19 & 0.81 & $0.63-0.99$ & $<0.001$ \\
\hline \multirow[t]{3}{*}{ MTHFR1298 } & AA & 484 & 48.45 & 1.55 & $1.51-1.59$ & \\
\hline & $\mathrm{AC}$ & 426 & 42.64 & 1.54 & $1.50-1.58$ & \\
\hline & $\mathrm{CC}$ & 89 & 8.91 & 1.44 & $1.35-1.53$ & 0.072 \\
\hline \multirow[t]{3}{*}{ MTHFR677 } & $\mathrm{CC}$ & 285 & 28.70 & 1.52 & $1.47-1.57$ & \\
\hline & $\mathrm{CT}$ & 491 & 49.95 & 1.55 & $1.51-1.59$ & \\
\hline & TT & 217 & 21.85 & 1.54 & $1.49-1.59$ & 0.708 \\
\hline \multirow[t]{3}{*}{ Tser } & 2R2R & 199 & 19.70 & 1.55 & $1.50-1.60$ & \\
\hline & 2R3R & 465 & 46.04 & 1.54 & $1.50-1.58$ & \\
\hline & 3R3R & 327 & 32.38 & 1.52 & $1.48-1.56$ & 0.587 \\
\hline \multirow[t]{5}{*}{ Cancer type } & colon & 549 & 54.36 & 1.51 & $1.48-1.54$ & \\
\hline & breast & 105 & 10.40 & 1.43 & $1.34-1.51$ & \\
\hline & gastric & 106 & 10.50 & 1.58 & $1.49-1.67$ & \\
\hline & pancreas & 62 & 6.14 & 1.62 & $1.53-1.71$ & \\
\hline & others & 188 & 18.61 & 1.52 & $1.42-1.63$ & 0.102 \\
\hline
\end{tabular}

*analysis of variance.

$* *$ for males $68 / 69 \mathrm{yrs}$; for females 64/65 yrs.

both activating (i.e.: orotate phosphoribosyltransferase, OPRT; thymidylate phosphorylase, TP) and inactivating (i.e.: dihydropyrimidine dehydrogenase, DPD) (Figure 1). Hence, the 5-FUDR parameter translates the effects of known and unknown genetic determinants, affecting 5-FU metabolizing enzymes, into a measurable phenotype. In this study, we have evaluated the general characteristics of the pretreatment 5-FUDR value on more than 1000 subjects, showing that it is a continuous parameter with a normal distribution in the population. This observation is consistent with the known existence of a fraction of persons with a very low rate of 5-FU metabolism (PM), but also highlights the existence of a class of subjects with an extremely rapid 5-FU metabolism (UM).

The effect of the individual 5-FUDR on 5-FU toxicity was analyzed in a subgroup of $433 \mathrm{CRC}$ patients. The total number of severe toxicities observed in this group ( $n=92,21.2 \%)$, as well as the higher frequency in older patients, are consistent with the data reported in the literature $[12,13]$.

Strikingly, we have found that both the PM subjects, defined by a 5 -FUDR $<=5$ th centile, and the UM subjects, defined by a 5-FUDR $>95$ th centile, are at higher risk of developing G3-4 toxicity, with an OR of 3.47 and 3.34, respectively, compared to the class of normal metabolizers $(5$ th $<5$-FUDR $<=95$ th centiles). While a higher percentage of toxicity in PM was expected, the association of 5-FU UM with severe toxicity was more surprising. Indeed, to our knowledge, no similar relationship between toxicity and a fast drug metabolism has been reported, probably due to the lack of analytical tests able to identify this kind of ultra-metabolizers. The potential clinical consequences of a 5-FU ultra-rapid metabolism are intriguing and deserve further studies. In fact, the mechanism underlying a faster rate of 5-FU consumption (that is, a higher 5-FUDR value) may involve an increased activity of DPD or an increased activity of the enzymes producing the active drug metabolites (Figure 1). This hypothesis is consistent with data showing that the 5-FU sensitivity is correlated with polymorphisms in the OPRT gene as well as in cancer tissues with the level of activity of the OPRT enzyme and with the OPRT/DPD activity ratio [29-32]. Thus, it could be speculated that the individual 5-FUDR may be related to progression free survival (PFS) and overall survival (OS), due to an increased fraction of cytotoxic metabolites in ultrametabolizers.

As reported in Table 3, in the analyzed CRC patients cohort, the 5-FUDR test allows risk stratification with a specificity and a NPV similar to that obtained using the 
Table 2: Association of severe toxicity (CTC3 Grade 3-4) with demographic, genotype, 5-FU degradation rate (5-FUDR) and chemotherapy characteristics, in the sample of colorectal cancer patients $(N=433)$ by univariate and multivariate analyses

\begin{tabular}{|c|c|c|c|c|c|c|c|c|c|c|}
\hline & & \multicolumn{2}{|c|}{ Total* } & \multicolumn{2}{|c|}{ Grade 0-2 } & \multicolumn{2}{|c|}{ Grade 3-4 } & \multirow[b]{2}{*}{$p$-value** } & \multirow[b]{2}{*}{ OR $^{\mathrm{a}}(95 \% \mathrm{CI})$} & \multirow[b]{2}{*}{$\mathrm{OR}^{\mathrm{b}}(95 \% \mathrm{CI})$} \\
\hline & & $N$ & $\%$ & $N$ & $\%$ & $N$ & $\%$ & & & \\
\hline \multirow[t]{2}{*}{ Sex } & males & 238 & 54.97 & 192 & 56.30 & 46 & 50.00 & & 1 & 1 \\
\hline & females & 195 & 45.03 & 149 & 43.70 & 46 & 50.00 & 0.281 & $1.29(0.81-2.04)$ & $1.47(0.88-2.45)$ \\
\hline \multirow[t]{2}{*}{$\begin{array}{l}\text { Age } \\
\text { category*** }\end{array}$} & $<=$ median & 205 & 47.34 & 173 & 50.73 & 32 & 34.78 & & 1 & 1 \\
\hline & $>$ median & 228 & 52.66 & 168 & 49.27 & 60 & 65.22 & 0.007 & $2.39(1.43-4.01)$ & $2.41(1.41-4.12)$ \\
\hline \multirow[t]{2}{*}{ DPYD } & GG & 427 & 98.61 & 338 & 99.12 & 89 & 96.74 & & 1 & 1 \\
\hline & GA & 6 & 1.39 & 3 & 0.88 & 3 & 3.26 & 0.113 & $3.80(0.75-19.14)$ & $4.78(0.71-32.19)$ \\
\hline \multirow[t]{3}{*}{ MTHFR 1298} & AA & 206 & 48.02 & 156 & 46.15 & 50 & 54.95 & & 1 & 1 \\
\hline & $\mathrm{AC}$ & 183 & 42.66 & 150 & 44.38 & 33 & 36.26 & & $0.69(0.42-1.12)$ & $0.53(0.29-0.98)$ \\
\hline & $\mathrm{CC}$ & 40 & 9.32 & 32 & 9.47 & 8 & 8.79 & 0.317 & $0.78(0.34-1.80)$ & $0.68(0.23-2.04)$ \\
\hline \multirow[t]{3}{*}{ MTHFR677 } & $\mathrm{CC}$ & 122 & 28.57 & 99 & 29.46 & 23 & 25.27 & & 1 & 1 \\
\hline & $\mathrm{CT}$ & 209 & 48.95 & 158 & 47.02 & 51 & 56.04 & & $1.39(0.80-2.42)$ & $1.26(0.64-2.49)$ \\
\hline & $\mathrm{TT}$ & 96 & 22.48 & 79 & 23.51 & 17 & 18.68 & 0.305 & $0.93(0.46-1.85)$ & $0.62(0.25-1.56)$ \\
\hline \multirow[t]{3}{*}{ TYMS TSER } & $2 \mathrm{R} 2 \mathrm{R}$ & 83 & 19.53 & 65 & 19.29 & 18 & 20.45 & & 1 & 1 \\
\hline & $2 \mathrm{R} 3 \mathrm{R}$ & 198 & 46.59 & 154 & 45.70 & 44 & 50.00 & & $1.03(0.55-1.92)$ & $1.01(0.52-1.99)$ \\
\hline & $3 \mathrm{R} 3 \mathrm{R}$ & 144 & 33.88 & 118 & 35.01 & 26 & 29.55 & 0.624 & $0.80(0.41-1.56)$ & $0.74(0.36-1.53)$ \\
\hline \multirow[t]{3}{*}{ 5-FUDR } & $\begin{array}{c}\mathrm{NM}(5-95 \\
\text { centile) }\end{array}$ & 393 & 90.76 & 318 & 93.26 & 75 & 81.52 & & 1 & 1 \\
\hline & PM $(<5$ centile $)$ & 19 & 4.39 & 10 & 2.93 & 9 & 9.78 & & $3.82(1.50-9.72)$ & $3.47(1.19-10.10)$ \\
\hline & UM (> 95centile) & 21 & 4.85 & 13 & 3.81 & 8 & 8.70 & 0.002 & $2.61(1.04-6.52)$ & $3.34(1.27-8.83)$ \\
\hline \multirow[t]{5}{*}{ Chemotherapy } & mFolfox6 & 177 & 40.97 & 133 & 39.12 & 44 & 47.83 & & 1 & 1 \\
\hline & Xelox & 46 & 10.65 & 33 & 9.74 & 13 & 14.13 & & $1.19(0.58-2.46$ & $1.26(0.57-2.82)$ \\
\hline & Capecitibine & 131 & 30.32 & 111 & 32.65 & 20 & 21.74 & & $0.54(0.30-0.98)$ & $0.35(0.18-0.69)$ \\
\hline & mFolfiri & 64 & 14.81 & 50 & 14.71 & 14 & 15.22 & & $0.85(0.43-1.68)$ & $0.83(0.40-1.71)$ \\
\hline & 5-fluorouracil & 14 & 3.24 & 13 & 3.82 & 1 & 1.09 & 0.126 & $0.23(0.03-1.83)$ & $0.17(0.02-1.77)$ \\
\hline
\end{tabular}

Abbreviations: OR, odds ratio; CI, confidence interval; NM, normal metabolism; PM, poor metabolism; UM, ultra metabolism.

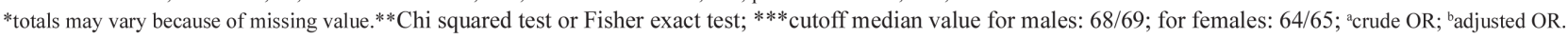

Table 3: Positive predictive value (PPV), negative predictive value (NPV), sensitivity and specificity of the 5-FU degradation rate (5-FUDR) test and the DPYD IVS14+1G> A genotyping test

\begin{tabular}{|l|c|c|c|c|}
\hline \multicolumn{2}{c}{ PPV } & NPV & Sensitivity & Specificity \\
\hline 5-FUDR PM+UM & 42.50 & 80.92 & 18.48 & 93.26 \\
\hline 5-FUDR PM & 47.37 & 80.92 & 10.71 & 96.95 \\
\hline 5-FUDR UM & 38.10 & 80.92 & 9.64 & 96.07 \\
\hline DPYD & 50 & 79.16 & 3.26 & 99.12 \\
\hline
\end{tabular}

Abbreviations: PM, poor metabolism; UM, ultra-rapid metabolism.

$D P Y D$ IVS $14+1 \mathrm{G}>$ A genotyping and with a PPV ranging from slightly to moderately lower than the DPYD IVS $14+1 \mathrm{G}>\mathrm{A}$ PPV $(47.37 \%$ for 5 -FUDR PM and $38.10 \%$ for 5 -FUDR UM compared to $50 \%$ of genotyping). In contrast, the sensitivity of the 5-FUDR test is much higher than the DPYD genotyping (18.48\% vs 3.26\%).). Even though it still is far from the ideal preemptive assay, this increase in sensitivity means that we would have had the opportunity to avoid14 more cases of severe (potentially lethal) toxicity compared to the 3 cases correctly predicted by the DPYD genotyping (Table 2). The advantage of the 5-FUDR test consists in the higher prevalence of the markers used to identify patients with poor and ultrarapid metabolism, which by definition consist of a $10 \%$ of the population (5-FUDR $<5$ th centile and $>95$ th centile) compared to the $1.28 \%$ frequency of the DPYD IVS $14+1 \mathrm{G}>$ A polymorphisms (frequency in the 1010 Italian patients sample).

However, proposal for novel preemptive tests must consider cost-effectiveness. In the case of 5-FUDR, the cost of the assay per sample is quite low (estimated at around \$10), since it does not required commercial kit but it is based on the chromatographic separation of the analyte, which is also a method easily transferrable into clinical laboratories. Considering that the cost derived 
by inpatient care of each G3-G4 toxicity is generally calculated in hundreds of dollars [33], the 5-FUDR assessment is supposed to be cost-effective. Further, the test result is available within one working day, so the test can easily be scheduled during the pre-treatment phase of patient evaluation and therapy selection.

A limitation for this study is that the pharmacogenetics assay for prediction of 5-FU toxicity includes only the main DPYD splice site polymorphisms, while additional functional polymorphisms have recently been proposed as important markers.

The future direction in the field of preemptive identification of severe toxicity during 5-FU treatment should point to the combination of genetic and phenotypic markers to improve the sensitivity for detection of patients at risk. The individual 5-FUDR appears as a suitable marker for this scope. Besides, the 5-FUDR test gives the opportunity to evaluate the potential clinical implications of the 5-FU ultra rapid metabolism and, in our opinion, it deserve further investigation as a prognostic markers of 5-FU treatment.

Finally, the significant number of severe toxicity currently undetectable by preemptive assays, highlights the need for a deeper understanding of the fluorouracil metabolism. Estimating the ratio between active and inactive drug metabolites probably will be the key to uncover multiple mechanisms mediating 5-FU efficacy and toxicities.

\section{MATERIALS AND METHODS}

\section{Patients}

Data collected from 1010 cancer patients followed at the Sant'Andrea Hospital of Rome, Italy, between April 2009 and April 2013, was analyzed in this retrospective study. Inclusion criteria were: age > 18 years; histologically documented cancer; Eastern Cooperative Oncology Group performance status 2 or less; cancer therapy toxicity absent; written informed consent. Exclusion criteria: relevant diseases within 6 months (i.e.: myocardial infarction, lung fibrosis, etc); 5-FU based chemotherapy in the past. Chemotherapy-related toxicity in the first six cycles of treatment was recorded according to National Cancer Institute Common Toxicity Criteria, version 3 (CTC3) [25].

To study associations between DNA polymorphisms, 5-FUDR and 5-FU-related toxicity, we have selected a subset of 433 CRC patients out of 1010 whose therapy was based essentially on fluorouracil, e.i.: mFOLFOX6, mFOLFIRI, XELOX with or without bevacizumab or cetuximab, and capecitabine.

The study was conducted in accordance with the Declaration of Helsinki and the protocol was approved by the institutional (Sapienza University) ethical committee (Rif. 3762_2015/23.07.2015, Prot. 2377/2015).

\section{Genotyping}

Germinal polymorphisms were analyzed as follows: genomic DNA was isolated from peripheral blood using the X-tractor Gene system (Corbett Life Science, Australia). The splice-site polymorphism, IVS14+1G $>$ A in the DPYD gene, C677T and A1298C SNPs in MTHFR gene were analyzed using the commercial kit for fluoropyrimidine response (Diatech, Jesi, Italy) according to manufacturer's protocol; briefly, region covering the SNP of interest was amplified by PCR using specific primers, and then sequenced using the Pyrosequencer PyroMark ID system (Biotage $\mathrm{AB}$ and Biosystems, Uppsala, Sweden). The variable number of tandem repeats (VNTR; 2R or 3R) in the TYMS enhancer region (TSER) was determined by the Polymerase Chain Reaction (PCR) according to manufacturer's protocol (fluoropyrimidine response - Diatech, Jesi, Italy) and visualized onto $2.2 \%$ agarose gel.

\section{Determination of the individual 5-FU degradation rate}

The test was performed as previously reported [23], using a HPLC-MS/MS instrument (high performance liquid chromatography-mass spectrometry/mass spectrometry) including an Agilent 1100 chromatographic system coupled to an API 3200 triple quadrupole (ABSCIEX, Framingham, MA, USA). Briefly, freshly prepared peripheral blood mononuclear cells $\left(2.5-3.5 \times 10^{6}\right.$ cells $)$ are incubate at $37^{\circ} \mathrm{C}$, with shaking, with a known amount of 5-FU. Cells aliquots are drawn at time $0,1 \mathrm{~h}$ and $2 \mathrm{~h}$, lysed and centrifuged and the concentration of 5-FU in the supernatants is quantified by HPLC-MS/MS. The 5-FUDR is expressed as ng $5-\mathrm{FU} / \mathrm{ml} / 10^{6}$ cells/min.

\section{Statistics}

Statistical analyses were performed using STATA, version 11.0 (StataCorp, College Station, Tex).

Shapiro-Wilk test was used as formal test for departure from a normal distribution, and histogram with a normal curve and a kernel density curve overlaid was also performed for visual inspection of 5-FUDR and age data distribution.

To remove some variability in outcome -at all covariate values-, while maintaining structure of the relationship between outcome and independent variables, the independent variables were categorized as follow. Subjects were subdivided into two groups with respect to median age. The 5-FUDR parameter was divided into 6 groups according with the value of the 5 th, 25 th, 50th, 75th, and 95th centile $(\leq 0.85 ;>0.85 \leq 1.25 ;>1.25$ $\leq 1.55 ;>1.55 \leq 1.84 ;>1.84 \leq 2.2 ;>2.2 \mathrm{ng} / \mathrm{ml} / 10^{6}$ cells/ min, respectively). For further analysis on the CRC 
patients subset, the data between 0.86 and $2.2 \mathrm{ng} / \mathrm{ml} / 10^{6}$ cells/min (5th-95th centiles) were taken together to create a reference group (NM, normal metabolisms). Dummy code was applied to sex, female $=1$ and male $=0$ in regression analysis.

Data about symptoms' severity were dichotomized as no/mild toxicities (CTC3 grade 0,1 and 2) versus severe toxicity (CTC3 G3-4).

Data, presented as proportions and differences between groups, were tested with Chi-squared or Fisher exact test. Univariate and multivariate odds ratios (ORs), and associated 95\% confidence intervals (CIs) for potential variables associated with severe toxicities were estimated using logistic regression models. We used the following model-building process: first we assessed bivariate associations between the dependent variable and each of the potential covariates; covariates not significantly associated $(p>0.10)$ with the outcome were dropped from further consideration in modeling outcome. The remaining candidate covariates were entered into a multiple regression model and subjected to backward selection until all remaining covariates had $p$-value $<0.05$, adjusted for the other remaining covariates. Gender, age, and gene polymorphisms were treated as confounding variables. Akaike's information criteria and the likelihood ratio test were used to define the multivariable model.

Deviations from Hardy-Weinberg equilibrium were assessed using the online HWE test calculator at http:// www.oege.org/software/hwe-mr-calc.shtml [24].

\section{Abbreviations}

5-FU: 5-fluorouracil; 5-FUDR: 5-FU degradation rate (expressed as $\mathrm{ng} 5-\mathrm{FU} / \mathrm{ml} / 10^{6}$ cells/min); CI: confidence interval; CRC: colorectal cancer; CTC3: National Cancer Institute Common Toxicity Criteria, version 3; DHFU: 5, 6-dihydrofluorouracil; DHP: dihydropyrimidinase; DPD: dihydropyrimidine dehydrogenase; DUT: dTUPase; FBAL: $\alpha$-fluoro- $\beta$ alanine; FdUDP: fluorodeoxyuridine diphosphate; FdUMP: fluorodeoxyuridine monophosphate; FdUTP: fluorodeoxyuridine triphosphate; FUDP: fluorouridine diphosphate; FUDR: 5-fluoro-2'-deoxyuridine; FUMP: fluoroyuridine monophosphate; FUPA: $\alpha$-fluoro- $\beta$ ureidopropionic acid;

FUR: 5-fluorouridine; FUTP: fluorouridine triphosphate; G3: grade 3 toxicity; G4: grade 4 toxicity;

HPLC: high performance liquid chromatography; MS: mass spectrometry; MTHFR: methylenetetrahydrofolate reductase; NM: normal metabolism; NPV: negative predictive value; OPRT: orotate phosphoribosyltransferase; OR: odds ratio; OS: overall survival; PBMC: peripheral blood mononuclear cells; PCR: Polymerase Chain Reaction; PFS: progression free survival; PM: poor metabolism; PPV: positive predictive value; QUASAR2: Quick and Simple and Reliable study; RRM2: ribonucleotide reductase; SD: standard deviation; TK: thymidine kinase; TP: thymidylate phosphorylase; TSER: thymidylate synthase enhancer region; TYMS: thymidylate synthase; U: uracil; UCK1: uridine diphosphate kinase; UH2: dihydrouracil; UK: uridine kinase; UM: ultra-rapid metabolism; UMPK: uridine monophosphate kinase; UPB: $\beta$-ureidopropionase; UPP1: uridine phosphorylase; VNTR: variable number of tandem repeat.

\section{CONFLICTS OF INTEREST}

Nothing to declare.

\section{REFERENCES}

1. Gustavsson B, Carlsson G, Machover D, Petrelli N, Roth A, Schmoll HJ, Tveit KM, Gibson F. A Review of the Evolution of Systemic Chemotherapy in the Management of Colorectal Cancer. Clin Colorectal Cancer. 2015; 14:1-10.

2. Wilson PM, Danenberg PV, Johnston PG, Lenz HJ, Ladner RD. Standing the test of time: targeting thymidylate biosynthesis in cancer therapy. Nat Rev Clin Oncol. 2014; 11:282-298.

3. Rich TA, Shepard RC, Mosley ST. Four decades of continuing innovation with fluorouracil: Current and future approaches to fluorouracil chemoradiation therapy. J Clin Oncol. 2004; 22:2214-2232.

4. Meta-Analysis Group in Cancer. Toxicity of fluorouracil in patients with advanced colorectal cancer: effect of administration schedule and prognostic factors. J Clin Oncol. 1998; 16:3537; 3541.

5. Schwartzberg LS, Vogel WH, Campen CJ. Methotrexate and Fluorouracil Toxicities: A Collaborative Practice Approach to Prevention and Treatment. The ASCO Post 2014; 5: Supplement.

6. Bamat MK, Tremmel R, Eberwine SF, Garcia R, Saydoff JA, von Borstel R. Uridine triacetate for prevention of 5-FU toxicity due to dihydropyrimidine dehydrogenase (DPD) deficiency. J Clin Oncol. 2011; 29:Supplement. ASCO Annual Meeting Abstracts Part1.

7. Boisdron-Celle M, Remaud G, Traore S, Poirier AL, Gamelin L, Morel A, Gamelin E. 5-Fluorouracil-related severe toxicity: a comparison of different methods for the pretherapeutic detection of dihydropyrimidine dehydrogenase deficiency. Cancer Letter. 2007; 249:271-282.

8. Longley DB, Harkin DP, Johnston PG. 5-fluorouracil: mechanisms of action and clinical strategies. Nat Rev Cancer. 2003; 3:330-338.

9. Ciccolini J, Mercier C, Dahan L, Evrard A, Boyer JC, Richard K. Toxic death-case after capecitabine + oxaliplatin (XELOX) administration: probable implication 
of dihydropyrimidine deshydrogenase deficiency. Cancer Chemother Pharmacol. 2006; 58:272-275.

10. Maring JG, van Kuilenburg AB, Haasjes J, Piersma H, Groen HJM, Uges DRA, Van Gennip AH, De Vries EGE. Reduced 5-FU clearance in a patient with low DPD activity due to heterozygosity for a mutant allele of the DPYD gene. Br J Cancer. 2002; 86:1028-1033.

11. Van Kuilenburg AB, Meinsma R, Zoetekouw L, Van Gennip AH. Increased risk of grade IV neutropenia after administration of 5-fluorouracil due to a dihydropyrimidine dehydrogenase deficiency: high prevalence of the IVS14+1g>a mutation. Int J Cancer. 2002; 101:253-258.

12. Schwab M, Zanger UM, Marx C, Schaeffeler E, Klein K, Dippon J, Kerb R, Blievernicht J, Fischer J, Hofmann U, Bokemeyer C, Eichelbaum M. German 5-FU Toxicity Study Group. Role of genetic and nongenetic factors for fluorouracil treatment-related severe toxicity: a prospective clinical trial by the German 5-FU Toxicity Study Group. J Clin Oncol. 2008; 26:2131-2138.

13. Gentile G, Botticelli A, Lionetto L, Mazzuca F, Simmaco M, Marchetti P, Borro M. Genotype-phenotype correlations in 5-fluorouracil metabolism: a candidate DPYD haplotype to improve toxicity prediction. Pharmacogenomics J. 2015; 28. doi: $10.1038 /$ tpj.2015.56.

14. Caudle KE, Thorn CF, Klein TE, Swen JJ, McLeod HL, Diasio RB, Schwab M. Clinical Pharmacogenetics Implementation Consortium guidelines for dihydropyrimidine dehydrogenase genotype and fluoropyrimidine dosing. Clin Pharmacol Ther. 2013; 94:640-645.

15. Marcuello E, Altes A, Menoyo A, Rio ED, Baiget M. Methylenetetrahydrofolate reductase gene polymorphisms: genomic predictors of clinical response to fluoropyrimidinebased chemotherapy? Cancer Chemother Pharmacol. 2006; 57:835-840.

16. Sharma R, Hoskins JM, Rivory LP, Zucknick M, London R, Liddle C, Clarke SJ. Thymidylate Synthase and Methylenetetrahydrofolate Reductase Gene Polymorphisms and Toxicity to Capecitabine in Advanced Colorectal Cancer Patients. Clin Cancer Res. 2008; 14:817-825.

17. Jakobsen A, Nielsen JN, Gyldenkerne N, Lindeberg J. Thymidylate synthase and methylenetetrahydrofolate reductase gene polymorphism in normal tissue as predictors of fluorouracil sensitivity. J Clin Oncol. 2005; 23:1365-1369.

18. Etienne-Grimaldi M-C, Francoual M, Formento JL, Milano G. Methylenetetrahydrofolate reductase (MTHFR) variants and fluorouracil-based treatments in colorectal cancer. Pharmacogenomics. 2007; 8:1561-1566.

19. Lu Z, Zhang R, Diasio RB. Dihydropyrimidine dehydrogenase activity in human peripheral blood mononuclear cells and liver: population characteristics, newly identified deficient patients, and clinical implication in 5-fluorouracil chemotherapy. Cancer Res. 1993; 53:5433-5438.

20. van Kuilenburg AB, van Lenthe H, Zoetekouw L, Kulik W. HPLC-electrospray tandem mass spectrometry for rapid determination of dihydropyrimidine dehydrogenase activity. Clin Chem. 2007; 53:528-530.

21. Ciccolini J, Mercier C, Evrard A, Dahan L, Boyer JC, Duffaud F, Richard K, Blanquicett C, Milano G, Blesius A, Durand A, Seitz JF, Favre R,et al. A rapid and inexpensive method for anticipating severe toxicity to fluorouracil and fluorouracil-based chemotherapy. Ther Drug Monit. 2006; 28:678-685.

22. Bi D, Anderson LW, Shapiro J, Shapiro A, Grem JL, Takimoto $\mathrm{CH}$. Measurement of plasma uracil using gas chromatography-mass spectrometry in normal individuals and in patients receiving inhibitors of dihydropyrimidine dehydrogenase. J Chromatogr B Biomed Sci Appl. 2000; 738:249-258.

23. Lostia AM, Lionetto L, Ialongo C, Gentile G, Viterbo A, Malaguti P, Paris I, Marchetti L, Marchetti P, De Blasi A, Simmaco M. A liquid chromatography-tandem mass spectrometry method for the determination of 5-Fluorouracil degradation rate by intact peripheral blood mononuclear cells. Ther Drug Monit. 2009; 31:482-488.

24. Rodriguez S, Gaunt TR, Day INM. Hardy-Weinberg equilibrium testing of biological ascertainment for Mendelian randomization studies. Am J Epidemiol. 2009; 169:505-514.

25. Common Terminology Criteria for Adverse Events (CTCAE), version 3. US National Cancer Institute. 2003.

26. Ruzzo A, Graziano F , Galli F Giacomini E, Floriani I, Galli F, Rulli E, Lonardi S, Ronzoni M, Massidda B, Zagonel V, Pella N, Mucciarini C, et al. Genetic markers for toxicity of adjuvant oxaliplatin and fluoropyrimidines in the phase III TOSCA trial in high-risk colon cancer patients. Sci Rep. 2014; 4:6828.

27. Rosmarin D, Palles C, Church D, Domingo E, Jones A, Johnstone E, Wang H, Love S, Julier P, Scudder C, Nicholson G, Gonzalez-Neira A, Martin M, et al. Genetic markers of toxicity from capecitabine and other fluorouracilbased regimens: investigation in the QUASAR2 study, systematic review, and meta-analysis. J Clin Oncol. 2014; 32:1031-1039.

28. Lee AM, Diasio RB. Genetic biomarkers for Fluorouracil toxicity prediction: the long road to clinical utility. J Clin Oncol. 2014; 32:989-990.

29. Tsunoda A, Nakao K, Watanabe M, Matsui M, Ooyama A, Kusano M. Associations of various gene polymorphisms with toxicity in colorectal cancer patients receiving oral uracil and tegafur plus leucovorin: a prospective study. Ann Oncol. 2011; 22:355-361.

30. Furuse H, Hirano Y, Harada M, Hong Ming L, Aoki T, Kurita Y, Mugiya S, Ushiyama T, Ozono S. Significance of 5-fluorouracil-related enzyme activities in predicting sensitivity to 5-fluorouracil in bladder carcinoma. Anticancer Res. 2009; 29:1001-1008.

31. Ochiai T, Umeki M, Miyake H, Iida T, Okumura M, Ohno K, Sakamoto M, Miyoshi N, Takahashi M, 
Tsumura H, Tokunuga Y, Naitou H, Fukui T. Impact of 5-fluorouracil metabolizing enzymes on chemotherapy in patients with resectable colorectal cancer. Oncol Rep. 2014; 32:887-892.

32. Sakamoto E, Nagase H, Kobunai T, Oie S, Oka T, Fukushima M, Oka T. Orotate phosphoribosyltrasferase expression level in tumors is a potential determinant of the efficacy of 5-fluorouracil. Biochem Biophys Res Commun. 2007; 363:216-222.
33. Delea TE, Vera-Llonch M, Edelsberg JS, McGarry L, Anton S, Ulcickas-Yood M, Oster G. The incidence and cost of hospitalization for 5-FU toxicity among Medicare beneficiaries with metastatic colorectal cancer. Value Health. 2002; 5:35-43. 